\title{
Regulative capacity of the cranial neural tube to form neural crest
}

\author{
Talma Scherson ${ }^{1}$, George Serbedzija ${ }^{2}$, Scott Fraser ${ }^{2}$ and Marianne Bronner-Fraser ${ }^{1, *}$ \\ ${ }^{1}$ Developmental Biology Center, University of California, Irvine, Ca. 92717, USA \\ ${ }^{2}$ Division of Biology, California Institute of Technology, Pasadena, Ca. 91125, USA \\ ${ }^{*}$ Author for correspondence
}

\section{SUMMARY}

In avian embryos, cranial neural crest cells emigrate from the dorsal midline of the neural tube shortly after neural tube closure. Previous lineage analyses suggest that the neural crest is not a pre-segregated population of cells within the neural tube; instead, a single progenitor in the dorsal neural tube can contribute to neurons in both the central and the peripheral nervous systems (Bronner-Fraser and Fraser, 1989 Neuron 3, 755-766). To explore the relationship between the 'premigratory' neural crest cells and the balance of the cells in the neural tube in the midbrain and hindbrain region, we have challenged the fate of these populations by ablating the neural crest either alone or in combination with the adjoining ventral portions of the neural tube. Focal injections of the vital dye, DiI, into the neural tissue bordering the ablated region demonstrate that cells at the same axial level, in the lateral and ventral neural tube, regulate to reconstitute a population of neural crest cells. These cells emigrate from the neural tube, migrate along normal pathways according to their axial level of origin and appear to give rise to a normal range of derivatives. This regulation following ablation suggests that neural tube cells normally destined to form CNS derivatives can adjust their prospective fates to form PNS and other neural crest derivatives until 4.5-6 hours after the time of normal onset of emigration from the neural tube.

Key words: cell lineage, ablation, regeneration, respecification, cell marking techniques, regulation, chick

\section{INTRODUCTION}

The vertebrate nervous system primarily arises from the neural plate, with the remaining contribution coming from the sensory epidermal placodes. At some point prior to or during neurulation, when the neural plate folds to form the neural tube, a divergence has been thought to occur between the central and peripheral nervous systems. While the former arises from the neural tube proper, the latter derives from neural crest cells at the junction of the neural plate and the lateral ectoderm. In avian embryos, neural crest cells emigrate from their origin at the dorsal aspect of the neural tube to migrate along well-defined pathways, settle in a variety of locations and differentiate into diverse cell types. Both the migratory pathways and the range of derivatives arising from neural crest cells are characteristic of their axial level of origin. For example, cranial neural crest cells give rise to elements of cranial ganglia as well as connective tissue, cartilage and bone of the face. Vagal (somites 1-7) neural crest cells contribute to the cardiac ganglion as well as much of the enteric nervous system. Trunk (somites 828) neural crest cells differentiate into sensory and autonomic ganglia, Schwann cells, chromaffin and other secretory cells, as well as melanocytes (LeDouarin, 1982).

The results of tissue grafts and ablations have demonstrated that some aspects of neural crest cell migration and differentiation are plastic, whereas others are somewhat fixed. For example, removal of the neural crest by the ablation of a small portion of the dorsal neural tube at the mesencephalic, occipital or cervical levels results in a normal embryo (McKee and Ferguson, 1984; Yntema and Hammond, 1947). This has been taken as evidence that neighboring neural crest cells, both rostral and caudal to the ablated region, repopulate the extirpated segments. In contrast, removal of any part of the vagal neural crest results in heart malformations (Kirby et al., 1983; Kirby, 1989), suggesting that this population in not regenerated after ablation. Grafting experiments of cells derived from various axial levels suggest a range of regulative abilities for the neural crest. For example, many cranial neural crest cells grafted to trunk axial levels assume normal trunk neural crest phenotypes (LeDouarin and Teillet, 1974). In contrast, when the neural crest cells that normally colonize the first branchial arch are grafted to a region where emerging neural crest cells populate the second branchial arch, the donor neural crest cells migrate into the foreign territory but give rise to structures appropriate for the first arch (Noden, 1983).

In the present series of experiments, we systematically examine the ability of the neural crest and neural tube cells to compensate for an ablation of premigratory cranial neural crest cells. Unlike previous ablation studies in which the origin of the compensating cells was unknown, we employed focal injections of the vital lipophilic dye, DiI, to label the tissue flanking either the rostral, caudal or ventral 
margins of the ablated region. The results show that the neural crest cells rostral or caudal to an ablated region display little or no compensation for the removed tissue. In contrast, the neural tube cells immediately ventral to the ablation clearly regulate to form a migratory cell population that gives rise to neural crest derivatives. This demonstrates that the divergence between the neural tube and neural crest is not absolute and remains capable of regulation until shortly after the onset of neural crest emigration.

\section{MATERIALS AND METHODS}

\section{Embryos}

White Leghorn chick embryos were incubated at $37^{\circ} \mathrm{C}$ until they reached the 4-12 somite stage (stages 8-11, Hamburger and Hamilton, 1951). A window was cut in the shell over the embryo and India Ink (Pelikan, Hanover, FRG) diluted 1:10 in Howard's Ringers solution was injected under the blastoderm to aid in visualization of the embryo. The vitelline membrane was deflected with a fine tungsten needle to allow access to the embryo.

\section{Ablations}

Segments of the premigratory neural crest either alone or in combination with part of the neural tube were removed unilaterally or bilaterally with glass needles. Glass capillary tubes were pulled to generate a sharp pointed tip. Incisions were made perpendicular to the long axis of the neural tube at the rostral and caudal edges of the site to be ablated. Longitudinal cuts were then made at both the boundary between the epidermis and the neural folds and the desired level within the neural tube. To avoid any possible contribution from the ablated tissue, it was removed from the egg by capillary action through a micropipette. Fig. 1 shows a schematic representation of the size and position of the ablations.

\section{Injection of vital dye}

Tissue adjacent to the ablated region was labelled by a focal injection of DiI (1,1-dioctadecyl-3,3,3',3'-tetramethylindocarbocyanine perchlorate; Molecular Probes) as described previously (Serbedzija et al., 1992). A $0.05 \%$ solution of DiI was prepared from a stock solution $(0.5 \%$ DiI in $100 \%$ ethanol) diluted $1: 10$ in $0.3 \mathrm{M}$ sucrose. Prior to use, the DiI solution was heated to $37^{\circ} \mathrm{C}$ to prevent cold shock to the embryos and to help keep the DiI in solution. For the double-labelling experiments, embryos received injections of both $\mathrm{DiI}$ and $\mathrm{DiO}$ (3,3'-dioctadecyloxacarbocyanine perchlorate; Molecular Probes) which was made up similarly to the DiI. The injection micropipettes (pulled to 10 $\mu \mathrm{m}$ tip from $1.2 \mathrm{~mm}$ o.d., $0.9 \mathrm{~mm}$ i.d., Al-Si filament glass capillary, A-M systems) were back filled with the DiI solution, attached to a Picospritzer II (General Valve) and mounted onto a micromanipulator (Marzhauser or Narashige). As schematized in Fig. 1 and illustrated in Fig. 2, a focal microinjection of DiI was made into the neural crest either immediately rostral or caudal to the ablated region, or into the edge of the neural tube immediately ventral to the ablated region. After dye injection, the egg was sealed with adhesive tape and returned to the incubator for 24-36 hours.

To verify the accuracy of the ablation as well as the precision of the DiI injection, a series of embryos $(n=5)$ was fixed immediately after the ablation and injection (Fig. 2A). Sections through these embryos showed a clean extirpation of the tissue between the residual neural tube and the overlying epithelium (Fig. 2B,C). The DiI-labelled cells were observed within a limited region of the neuroepithelium, immediately adjacent to the cut edge. The DiIlabelled cells were contained within 2 to 3 sections (approximately $40 \mu \mathrm{m})$.

\section{Confocal microscopy}

DiI-labelled embryos were viewed in whole mount using a laser scanning confocal microscope fitted with an argon-krypton laser (Biorad MRC 600 on a Zeiss Axiovert). A rhodamine filter set was used to visualize the $\mathrm{DiI}$; a rhodamine/fluorescein double-label filter set $(\mathrm{K} 1, \mathrm{~K} 2)$ was used to visualize the DiI and DiO. Raw data files were later transferred to a Macintosh IIci for processing with Adobe Photoshop (superimposing the DiI fluorescence image in red with the transmitted light image in blue-gray) and printing with a Nikon printer.

Based on the whole-mount images of the embryos, schematic diagrams were made summarizing the results of each class of operation (Figs 4, 5, 7 A'-D'). Each diagram shows a shaded region that represents the full range of labelled cells that migrated from the indicated injection site (each shaded region represents the data from six to twenty experimental cases). Because the position of the DiI injection in the neural tube is visible both at the time of injection and at the time of analysis, its position at the time of analysis could be used to group identically treated cases. This permitted us to compensate for slight rostrocaudal variations at the time of the experiment. Some schematics show data from more than one injection site to give an indication of this variation.

\section{Cryostat sections}

The distribution of the labelled cells, originally observed in whole mount preparations of embryos analyzed by confocal microscopy, was confirmed in frozen sections. Cross sections were analyzed on a conventional epifluorescence microscope. Embryos were fixed overnight in $4 \%$ paraformaldehyde $/ 0.25 \%$ glutaraldehyde at $4{ }^{\circ} \mathrm{C}$, rinsed and incubated in phosphate-buffered saline (PBS) for 2-3 hours, placed in $5 \%$ sucrose in PBS containing $0.01 \%$ sodium azide for 2-4 hours and into $15 \%$ sucrose in PBS at $4{ }^{\circ} \mathrm{C}$ overnight. The tissue was embedded in $7.5 \%$ gelatin (Sigma; 300 Bloom) in $15 \%$ sucrose/PBS at $37^{\circ} \mathrm{C}$ for 3-4 hours, oriented and frozen in liquid nitrogen. $20 \mu \mathrm{m}$ frozen sections were cut on a Zeiss Microm cryostat (Carl Zeiss Instruments), and mounted with Gel/mount (Biomeda) to prevent spreading of the dye. Sections were observed within 1-2 days. Some slides were stained with neurofilament antibodies, as described in Sechrist et al. (1993).

\section{Analysis of mitotic activity}

After ablation, embryos were allowed to recover for $1,3.5$ or 5.5 hours prior to application of $60 \mu \mathrm{l}$ of $10^{-6} \mathrm{M}$ solution of BrdU (bromodeoxyuridine; Sigma) for 1 hour in ovo. The embryos then were removed from the egg, washed, fixed in $70 \%$ ethanol at $4^{\circ} \mathrm{C}$ overnight and processed for cryostat sectioning as described above. Sections were treated with $2 \mathrm{~N} \mathrm{HCl}$ for 15-30 minutes, then washed with phosphate-buffered saline (PBS). The BrdU was detected using an anti-BrdU antibody (Becton-Dickinson) diluted 1:30 in PBS/BSA. Sections were viewed under the fluorescence microscope.

\section{RESULTS}

To test the regulative ability of the neural crest and neural tube in the midbrain (mesencephalon) and the hindbrain (rhombencephalon), we surgically ablated either the cranial neural folds or the neural folds plus most of the adjoining ventral neural tube (Fig. 1). The operation cleanly eliminated the desired region of the neurectoderm, leaving the rest of the embryo unperturbed (Fig. 2). The ablations were extensive, encompassing the neural folds along the entire length of the midbrain and/or the rostral portion of the hindbrain. Embryos ablated at the 4-7 somite stage and 
A
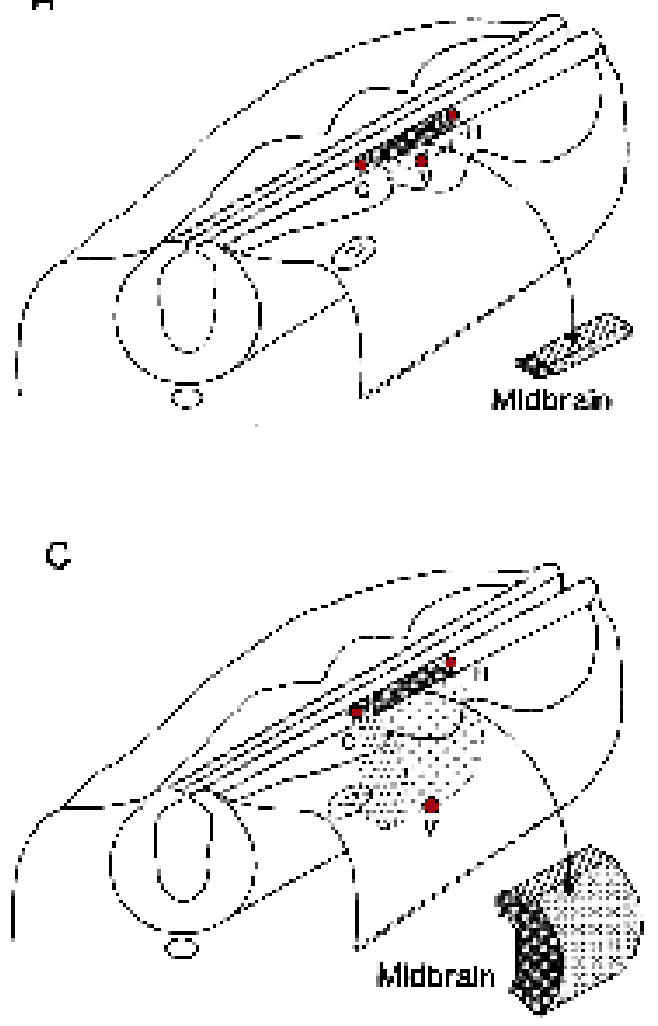

B

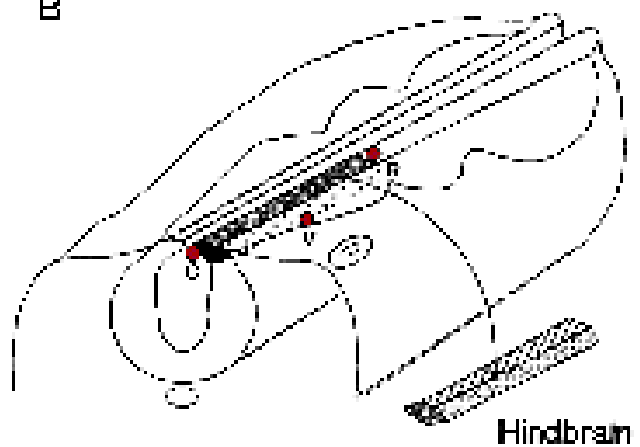

D

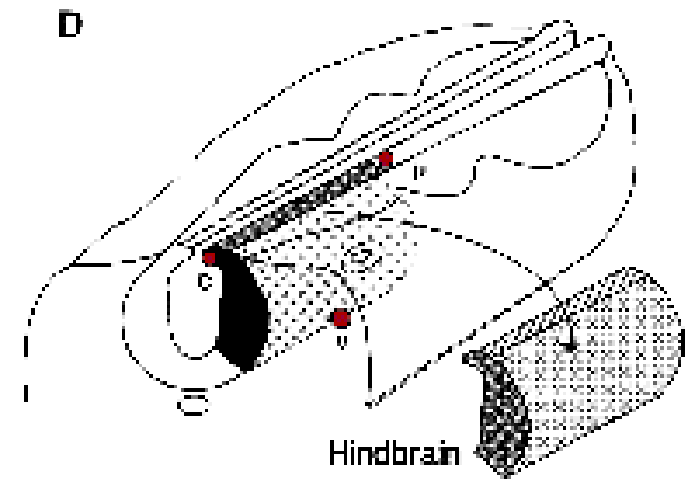

Fig. 1. Schematic representation of the microsurgical procedures. Ablations of the neural folds alone $(A, B)$ or of the neural folds plus the neural tube $(\mathrm{C}, \mathrm{D})$ were performed unilaterally or bilaterally with glass needles. The ablations extended over either the presumptive midbrain $(\mathrm{A}, \mathrm{C})$ or hindbrain (B,D), except in the case of the extensive ablations where both regions were removed. After the ablation, the neural tissue flanking the ablation rostrally (R), caudally (C) or ventrally $(\mathrm{V})$ was labelled with a focal injection of DiI.

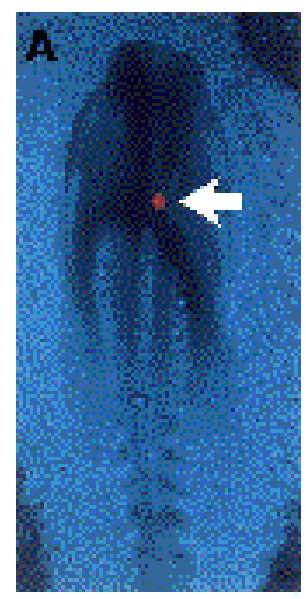

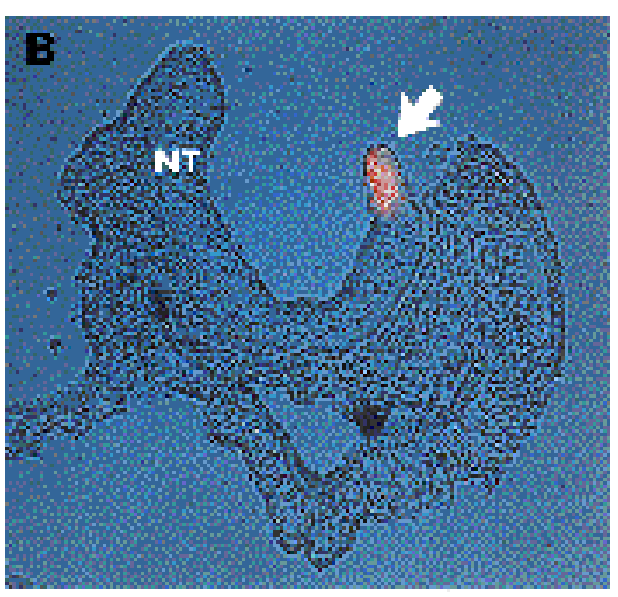

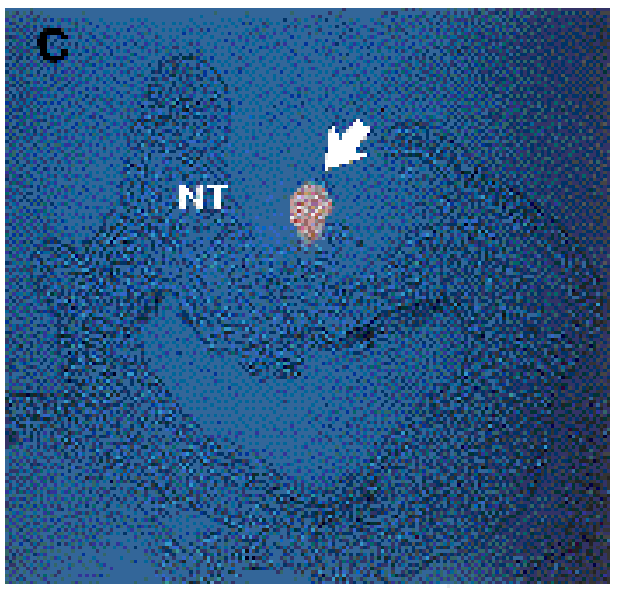

Fig. 2. Ablation of neural fold and/or neural tube followed by focal injections of neighboring neural tube cells with DiI. (A) A whole-mount view of a stage 8-9 embryo fixed shortly after the unilateral ablation of the midbrain neural crest and a focal DiI injection (red, arrow) into the neural tube ventral to the cut edge (as shown in Fig. 1A). (B) In a transverse section of the embryo pictured in A, the DiIlabelled cells (arrow) were confined to the neural tube (NT). (C) A transverse section through another embryo fixed shortly after a deep ablation of the midbrain neural folds plus neural tube (Fig. 1B); the neural tube ventral to the ablation was labelled with DiI (arrow). As in B, only a small population of the adjoining neural tube cells (NT) were labelled. 
allowed to develop in ovo from 1 to 7 days were morphologically similar to unoperated controls when viewed as whole mounts (Fig. 3A). Furthermore, histological sections of these embryos showed the presence of normal-appearing neural crest-derived ganglia and branchial arches (Fig. 3B). This strikingly normal development of neural crest derived structures after the elimination of their normal precursors in the neural folds demonstrated clearly that some form of regulation had taken place.

To explore the nature of this regulative response, each embryo received a focal injection of DiI adjacent to the rostral, caudal or ventral cut edges of the ablated region immediately after the operation (Fig. 1). These focal injections resulted in a single patch of DiI-labelled cells with a diameter of less that $40 \mu \mathrm{m}$ (Fig. 2). To determine which cells were altering their fate to compensate for the elimination of neural crest, the behavior of the labelled cells was compared with that of similarly labelled cells in unoperated embryos (see 'Fate Map' below). To facilitate such comparisons, Figs 4, 5 and 7 present both images of individual embryos and schematic diagrams illustrating the distribution of DiI-labelled cells for each type of operation.

\section{Fate map of normal cranial neural crest development}

To create a fate map against which the results of our perturbation experiments can be compared directly, focal injections of DiI were made into the neural folds and neural tube of unoperated embryos at the level of the caudal forebrain (prosencephalon), midbrain (mesencephalon) or hindbrain (rhombencephalon). The focal injections were similar to those applied previously to the chick (Lumsden et al., 1991; Sechrist et al., 1993) and the mouse embryo (Serbedzija et al., 1992). DiI injections into the dorsal neural tube (presumptive neural crest) at any axial level resulted in labelled cells distributed through predictable regions of the periphery. In all cases, some labelled cells remained in the neural tube, permitting an unambiguous identification of the site of labelling. Because slight differences in the exact position of the injection pipette can label distinct regions of the neuraxis, comparisons were made only between animals with identically positioned labelling sites in the neural tube.

Injection of DiI into the neural folds at the forebrain/midbrain border resulted in labelled cells coursing toward the frontonasal process and the rostral portion of the eye (Fig. 4A). Labelled cells emigrating from injection sites in the midbrain neural folds appeared as a dispersed stream oriented rostrolaterally, extending toward the peri-optic mesenchyme and maxillary process (Fig. 5A).

Injections into the hindbrain often were performed prior to the formation of all rhombomere boundaries; however, the exact position of each injection could be inferred in retrospect from the pattern of the DiI-labelled cells that remained within the neural tube at the time of fixation. Focal DiI injections into rhombomere 1 (r1) resulted in cells distributed similarly to those observed from the midbrain, plus an additional group of cells contributing to the first branchial arch. Injections into $r 2$ resulted in labelled neural crest cells that populated the first branchial arch. Thus, there is some overlap in the distribution of neural crest cells arising from the midbrain and rostral hindbrain. However, neural crest cells arising caudal to $\mathrm{r} 2$ display characteristic and relatively non-overlapping patterns of migration. For example, DiI injections into rhombomere 4 populated the second branchial arch (Fig. 4B) whereas those into rhombomere 6
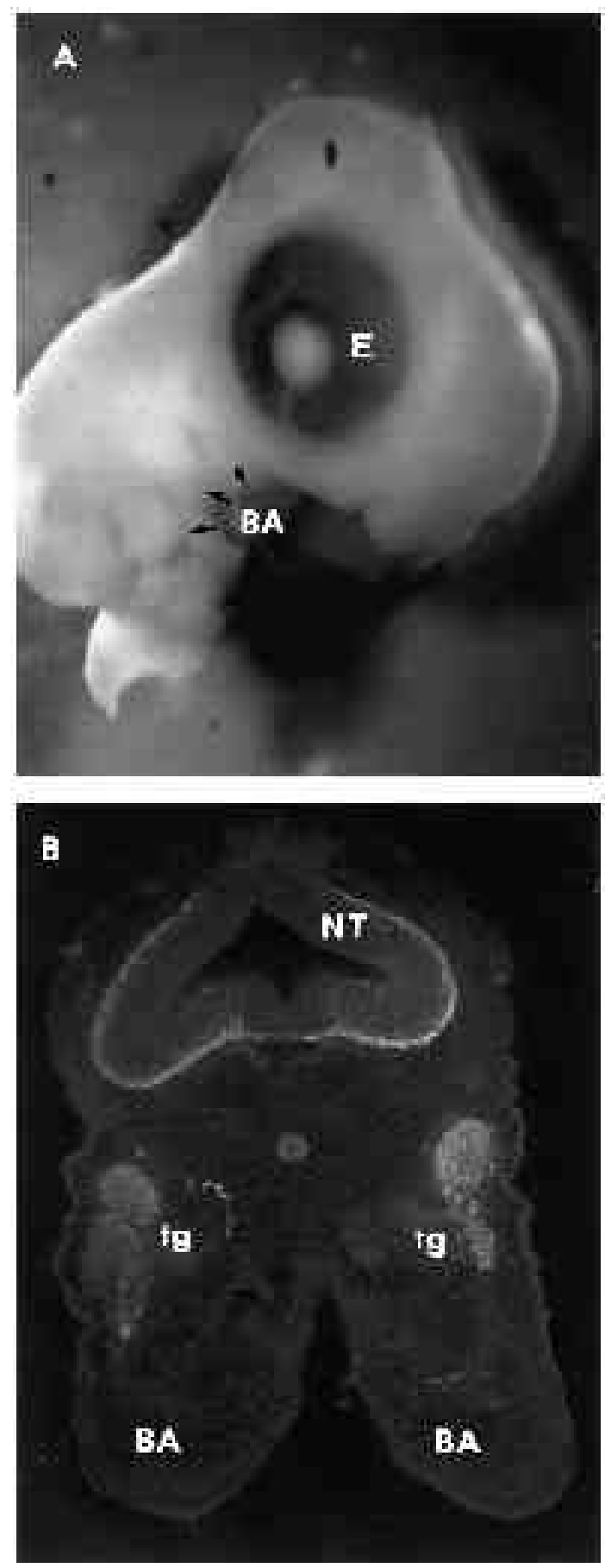

Fig. 3. Normal development after cranial neural crest ablation. (A) An embryo that developed in ovo for 5 days following unilateral neural fold ablation. The facial structures, many of which arise from the neural crest, were morphologically normal and indistinguishable from those in unoperated embryos. (B) In an anti-neurofilament-stained transverse section through an embryo fixed 2 days after ablation, the neural tube (NT), trigeminal ganglia (tg) and branchial arches (BA) appeared similar on both the operated (right) and unoperated side. 
populated the third branchial arch (see Lumsden et al., 1991 and Sechrist et al., 1993).

In contrast to labelling of the neural folds, injections of DiI into the ventral regions of the neural tube either in the midbrain (Fig. 5B, $\mathrm{B}^{\prime}$ ) or in the hindbrain (Figs 7A,B, 9A, A') resulted in brightly labelled cells exclusively within the neural tube. Thus, only neural tube cells at the dorsal margin contribute to migratory neural crest cells.

\section{Regulation by the adjoining rostral and caudal neural crest}

The apparently complete compensation for the ablated neural crest might result from neural crest cells from more rostral or more caudal axial levels being recruited to the ablated levels. To test this possibility, focal injections of DiI were performed into the neural folds bordering the rostral or caudal margin of the ablated neural folds (schematized in Fig. 1) and their pathways of migration were compared with the normal fate map.

In embryos examined 36 hours after extirpation of the neural folds in either the midbrain or hindbrain, the DiIlabelled neural crest cells from levels rostral or caudal to the ablation were observed exclusively along their normal pathways. They did not deviate to occupy the pathways normally followed by neural crest cells arising from the ablated region. For example, when the midbrain neural folds were ablated, DiI-labelled cells bordering the caudal edge (midbrain/hindbrain border) moved to the maxillary process and first branchial arch (Fig. 4D'i), just as cells in unoperated controls (Fig. 4B'i). Similarly, those emerging adjacent to the rostral cut edge (forebrain/midbrain border) moved to the frontonasal process (data not shown) as in unoperated embryos. When the hindbrain neural folds were ablated, DiIlabelled cells emerging from rostral to the cut edge (midbrain/hindbrain border) still moved to the maxillary process and first branchial arch; those emerging from caudal injections (around the level of rhombomere 4) invaded the second branchial arch (data not shown), just as they would in the unperturbed embryo (Lumsden et al., 1991; Sechrist et al., 1993). Thus, the DiI-labelled cells arising from the hindbrain of ablated embryos do not migrate to more rostral regions as would be expected if they were compensating for the removed neural crest.

Because the neighboring neural crest failed to compensate after ablations of either the midbrain or hindbrain neural folds, we performed a more extensive ablation of both midbrain and hindbrain tissues in the hope of creating a more extreme effect on the bordering neural crest cells. The ablation of the neural folds from both the midbrain and hindbrain (down to and including rhombomere 4) failed to alter the migration patterns of the bordering neural crest cells. Labelled cells rostral to the extensive ablation formed a stream that coursed past the rostral portion of the eye and toward the frontonasal primordium (Fig. 4C). For ablations extending from the midbrain to the level of rhombomeres $1 / 2$, labelled cells from caudal to the ablation were observed moving laterally through the mesenchyme and into the first branchial arch. For ablations extending from the midbrain to the level of rhombomeres $3 / 4$, labelled cells from caudal to the ablation moved into the second branchial arches (Figs $\left.4 \mathrm{D}^{\prime} \mathrm{ii}, 8\right)$. Thus, the patterns of migration were indistin- guishable from those of normal embryos (compare with Fig. $4 \mathrm{~A}^{\prime}$ and $\mathrm{B}^{\prime}$, respectively).

These labelling studies show conclusively that the appearance of normal neural crest derivatives after ablations of the midbrain and/or hindbrain neural folds, is not due to cells from more rostral and caudal levels migrating to fill in the ablated cells. These observations are even more surprising given that about $15 \%$ of the embryos receiving extensive ablations suffered defects in their vascular system, having excessive blood in the face and around the heart.

\section{Regulation by the adjoining ventral neural tube}

Given that the compensation for the missing cells is not from the neural crest cells rostral or caudal to the ablation, it may result from the neural tube cells that lie immediately ventral to the ablation. To test this possibility, focal injections of DiI were performed into the ventrolateral neural tube following neural fold ablation (Figs 1, 2), which normally do not give rise to any neural crest cells (Figs 5B, 7). Surprisingly, the neural tube injections resulted in labelled migratory cells (Fig. 5C,D) that moved in a pattern indistinguishable from that of normal neural crest cells at the corresponding axial levels (Fig. 5A). For example, labelled cells derived from the midbrain neural tube were observed in the cranial mesenchyme as a broad stream moving laterally toward the perioptic mesenchyme and maxillary process (Fig. 5C') identical to that of normal neural crest cells at that level (Fig. 5A'). Similarly, after neural fold ablation in the hindbrain, injections of DiI into the ventral neural tube resulted in labelled cells distributed much like the neural crest cells normally emerging from these levels (data not shown; Fig. 4B). Thus, it seems that the underlying neural tube cells regulate to form neural crest cells following the elimination of the pre-existing neural crest.

To determine if this regulative capacity extends to the most ventral regions of the neural tube, we ablated the neural tube unilaterally from the neural folds nearly to the ventral midline, but excluding the floor plate. In embryos fixed shortly after such an extensive ablation, a large gap was observed between the residual neural tube and the overlying epithelium (Fig. 2C). As little as 5 hours after ablation, some DiI-labelled cells emerging from the ventral cut edge of the neural tube appeared to be dispersing and migrating into the cranial mesenchyme (Fig. 6) even prior to 'reclosure' of the neural tube. This suggests that the fate of the remaining neural tube cells is rapidly readjusted after the ablation and that regulation of the neural crest may occur prior to reformation of a complete, normal-sized neural tube. When ablated embryos were labelled with BrdU as described in Materials and Methods, no dramatic increase in mitotic activity was apparent between 1 and 5.5 hours following the operation (data not shown). To test if the regenerated neural crest cells were HNK-1 immunoreactive, embryos were sectioned and stained with the HNK-1 antibody 1 day following injection. DiI-labelled cells were observed within streams of HNK-1 positive cells. Because the DiI signal was extremely bright, it made unequivocal identification of double-labelled cells difficult. However, a few cells were clearly both DiI and HNK-1 positive (data not shown). Therefore, at least some of the reconstituted neural crest cells emerging from the ventral neural tube are not only 
behaving in a fashion appropriate for the neural crest, but also express the HNK-1 antigen, typically present on migrating neural crest cells.

In embryos examined 36 hours after unilateral ablation of most of the midbrain neural tube, labelled cells from the ventral portion of the neural tube were observed migrating through the mesencephalic mesenchyme and populating the area around the eye and maxillary process (Fig. 5D). This pattern of migration was indistinguishable from that observed in unoperated control embryos after DiI labelling of midbrain neural folds (Fig. 5A). In contrast, injections of DiI into identical locations in unoperated embryos yielded groups of labelled cells confined within the ventral neural tube in the midbrain (Fig. 5B) or in the hindbrain (Figs 7 , $\left.9 \mathrm{~A}^{\prime}\right)$. Cryostat sections of embryos that had been injected in the rostral midbrain, caudal midbrain and r3 and r4 gave identical results to those of Fig. 7.

In order to compare, within single embryos, the respec-
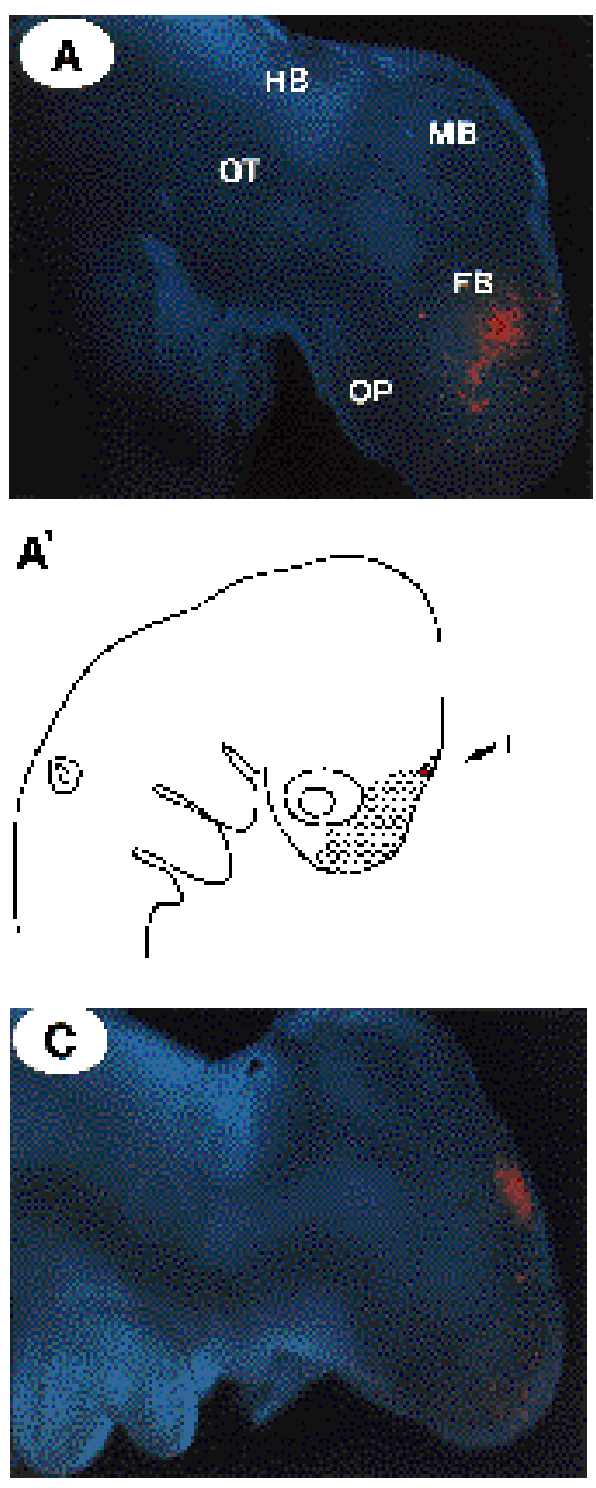

$\mathbf{C}^{\prime}$

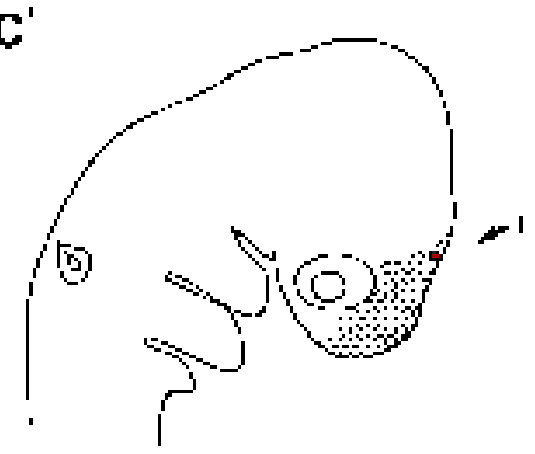

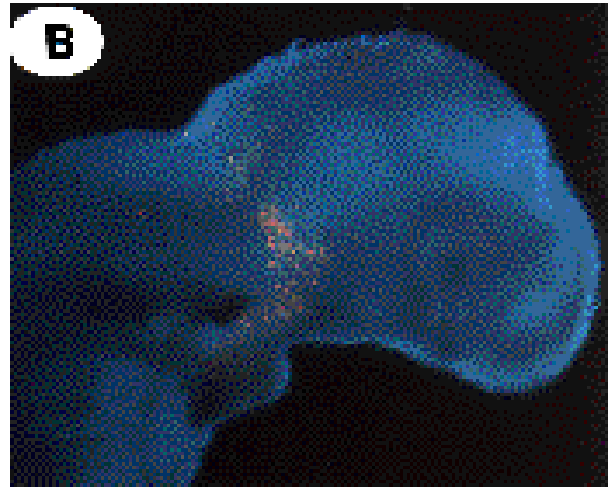

B'
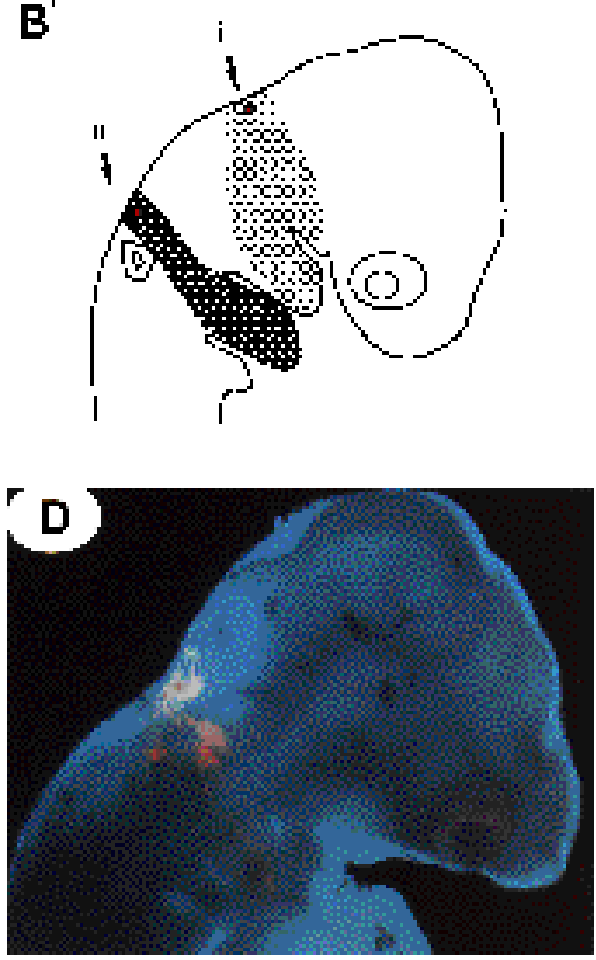

D'

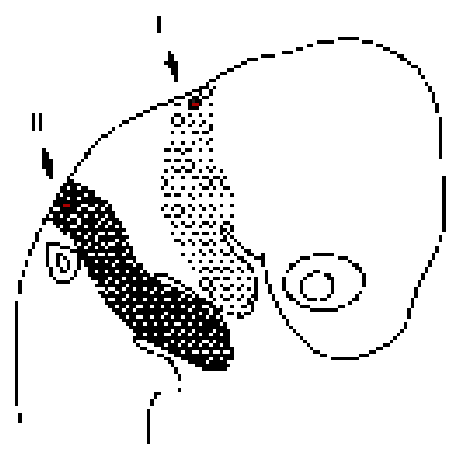

Fig. 4. Cells rostral and caudal to an ablation do not compensate for the missing neural crest or neural folds. For each class of operation, both a photograph of a representative embryo and a schematic diagram (') summarizing at least six cases are depicted. (A) In unoperated embryos, labelled by focal injection of DiI at the forebrain/midbrain border, labelled cells were observed coursing toward the frontonasal process and the rostral portion of the optic cup. The optic cup (OP), forebrain (FB), midbrain (MB), hindbrain (HB) and otic vesicle (OT) are indicated for reference. (B) Unoperated embryo labelled by a focal injection of DiI in the hindbrain neural folds. Labelled cells arising from rhombomere 2 (i) had migrated ventrolaterally into the first branchial arch; whereas those arising from the level of rhombomere $4 / 5$ (ii) contributed to the second branchial arch. (C) Following unilateral neural fold ablation in the midbrain and hindbrain, focal injection of DiI into the neural folds immediately rostral to the cut edge resulted in labelled cells in the frontonasal process and the peri-optic mesenchyme. For cases with equivalent injection sites, the distribution of labelled cells was indistinguishable from unoperated controls (compare $\mathrm{A}^{\prime}$ and $\mathrm{C}^{\prime}$ ).

(D) After ablations at the level of the midbrain alone or in combination with the hindbrain, the neural crest cells immediately caudal to the ablation migrated normally. Cells bordering a midbrain ablation migrated ventrolaterally to populate the first branchial arch $\left(\mathrm{D}^{\prime} \mathrm{i}\right)$; those bordering a midbrain/hindbrain ablation were observed within the second branchial arch (D'ii). 
tive contributions of the neural tube cells ventral to the ablation and the unperturbed dorsal neural fold cells caudal to the ablation, we performed double labelling with DiI and DiO. After the majority of the neural tube was ablated unilaterally in the midbrain and rostral hindbrain, the embryos received a focal injection of $\mathrm{DiO}$ into the midbrain neural tube and a focal injection of DiI into the neural folds just caudal to the ablation at the level of r4. As seen in Fig. 8, the neural tube cells (green) have dispersed laterally just as the normal neural crest cells in the midbrain. In contrast, preexisting neural crest cells (reddish-orange) migrated laterally and entered the second branchial arch, as expected for cells arising from $\mathrm{r} 4$. Only a few scattered cells deviated rostrally from their original trajectory (Fig. 8).

To assess whether there was any compensation from the contralateral neural crest after unilateral ablation of the
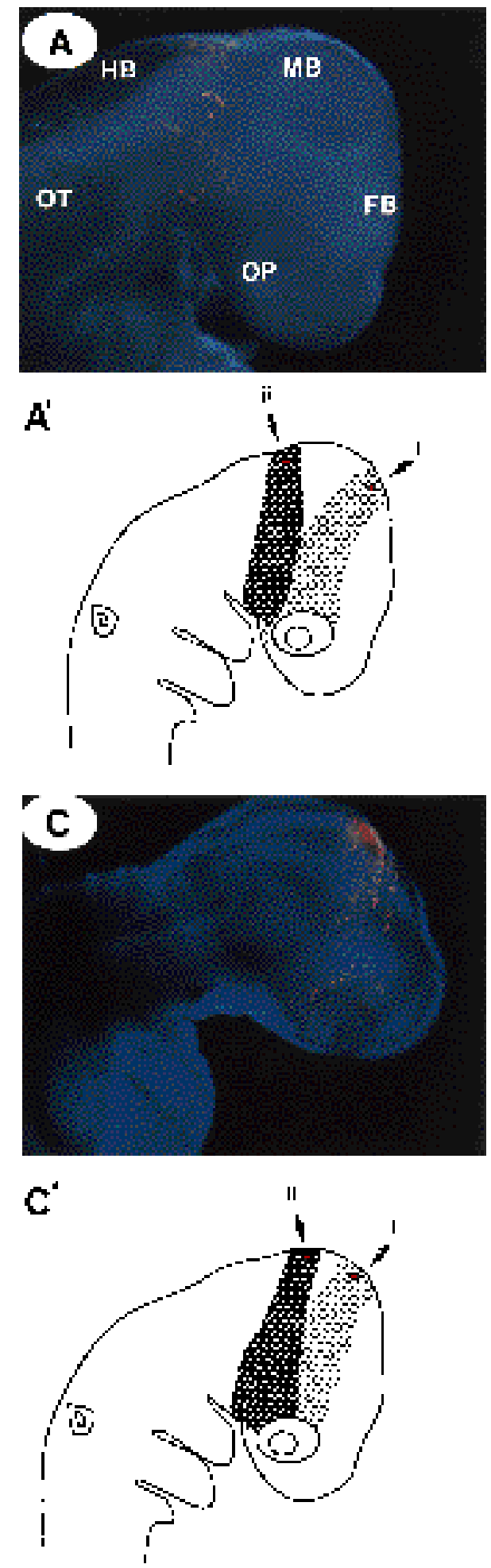

D'
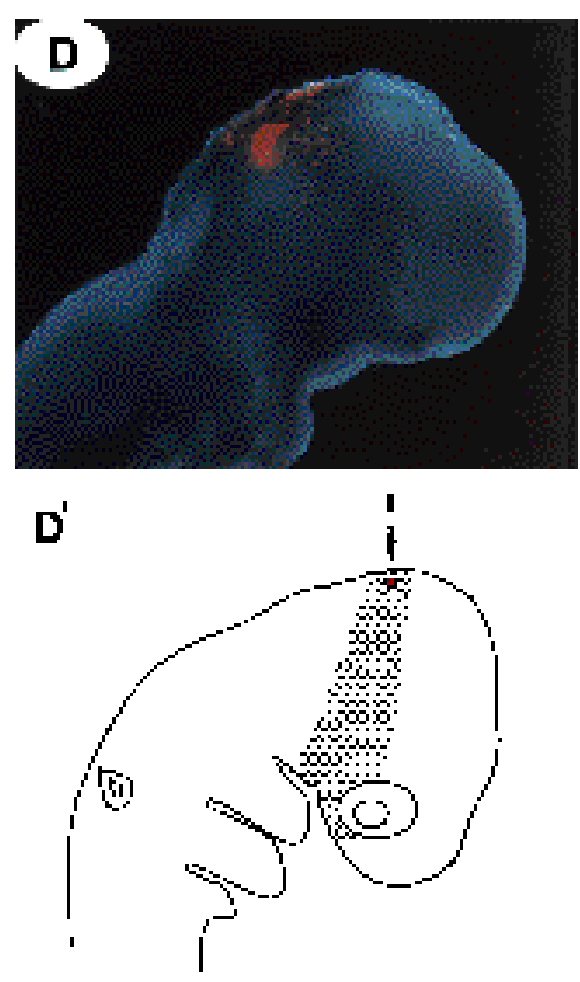

Fig. 5. Midbrain ventral neural tube forms neural crest after ablation of the cells dorsal to it. For each class of operation, both a photograph of a representative embryo and a schematic diagram (') summarizing at least six cases are depicted. (A) Unoperated embryos were labelled by focal injection of DiI in the dorsal neural tube at the level of the midbrain. Depending upon the exact level of the injection, labelled neural crest cells were observed in a stream that extended laterally to surround the optic cup (A'i) and/or the maxillary process (A'ii). The optic cup (OP), forebrain (FB), midbrain (MB), hindbrain (HB) and otic vesicle (OT) are indicated for reference. (B) In unoperated embryos that received a focal injection of DiI into the ventral neural tube, labelled cells were confined to a small region within the ventral neural tube. (C) After unilateral ablation of the neural fold, DiI-labelled cells from the immediately ventral neural tube migrated in a pattern similar to that of the neural crest in unoperated controls $\left(\mathrm{A}^{\prime}\right)$. The cells were distributed in a stream that extended toward the peri-optic mesenchyme and/or maxillary process depending upon the exact rostrocaudal position of the dye injection (i, ii). (D) After deep unilateral ablations of both the neural folds and the bulk of the neural tube, DiI-labelled cells immediately ventral to the ablation migrated away from the neural tube. Unlike the normal pattern observed after labelling the ventral neural tube in unoperated animals (B'), the labelled cells formed streams that extended laterally into the peri-optic mesenchyme, maxillary process or first branchial arch. 

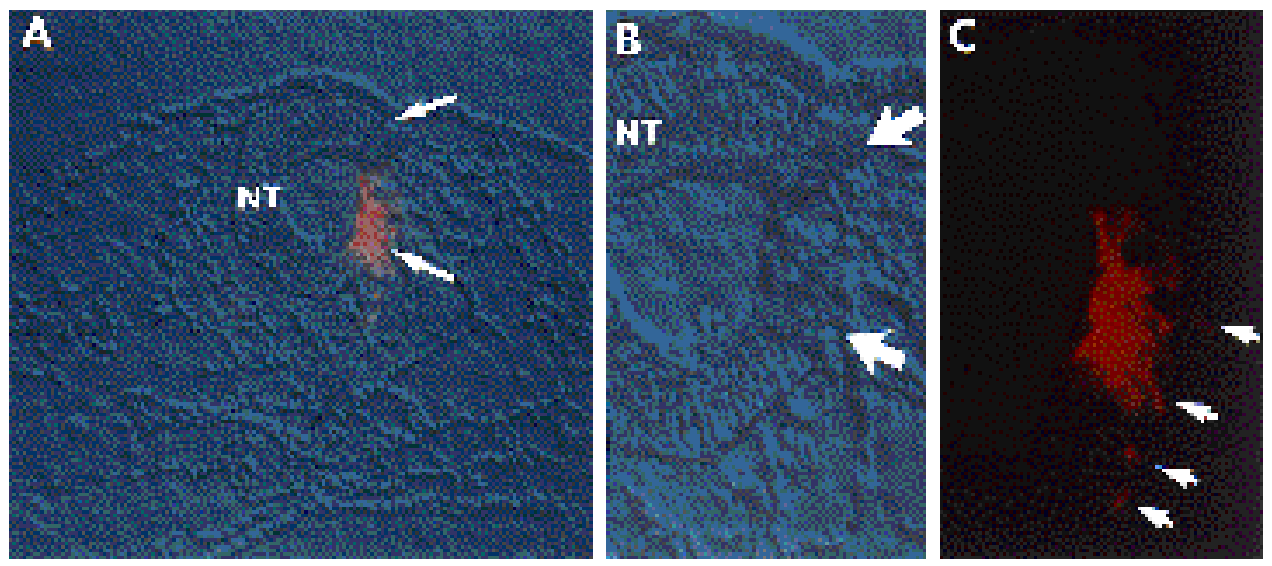

Fig. 6. Neural crest cells emigrate from the cut edge of the neural tube prior to tube reclosure. In an embryo examined 5 hours after unilateral ablation and focal injection of DiI into the ventral neural tube, the neural tube has not yet reclosed, but individual DiIlabelled cells can be observed the adjacent mesenchyme. (A) Phase plus fluorescence image illustrating the morphology of the neural tube (NT) and the position of the DiI label. The arrows indicate the position of the cut edges. Note that the neural tube from the contralateral side has shifted ipsilaterally. DiI-labelled cells are apparent in the neural tube as well as in the mesenchyme. (B) A higher magnification phase image in which the cut edges (arrows) are more clearly visible. (C) The fluorescent image of B illustrating that several individual DiI-labelled cells (small arrows) have emigrated into the cranial mesenchyme.

neural tube, we performed focal injections of DiI into the neural folds opposite the ablated side. In half of the cases (7/14), we observed some contribution from the contralateral neural crest. This is not surprising, since the descendants of single, labelled neural crest cells tend to distribute bilaterally (Bronner-Fraser and Fraser, 1988). In the 7 embryos in which we observed no contribution from the contralateral neural folds, the dorsal neural tube appeared to have prolapsed such that the labelled cells were located within the lumen of the neural tube (data not shown). The positioning of the labelled cells may account for their failure to contribute to the contralateral neural crest.

Similar results were obtained following the unilateral removal of the neural tube at the level of the hindbrain or of both the hindbrain and midbrain. In all cases, a morphologically complete neural tube was re-created; normal neural tube and neural crest morphologies were present by 36 hours. Labelled cells were observed in a stream that coursed laterally into the branchial arches (Fig. 9B). In contrast, injections of DiI into identical locations in unoperated embryos yielded a confined group of labelled cells within the ventral neural tube in the hindbrain (Fig. 9A).

Because all of the ablations described above were unilateral, it seemed possible that the integrity of the contralateral neural tube is required for regulation of the neural crest. To test this possibility, we ablated the neural folds and tube bilaterally, leaving only the ventral midline region intact. Then, focal injections of DiI were performed either into the
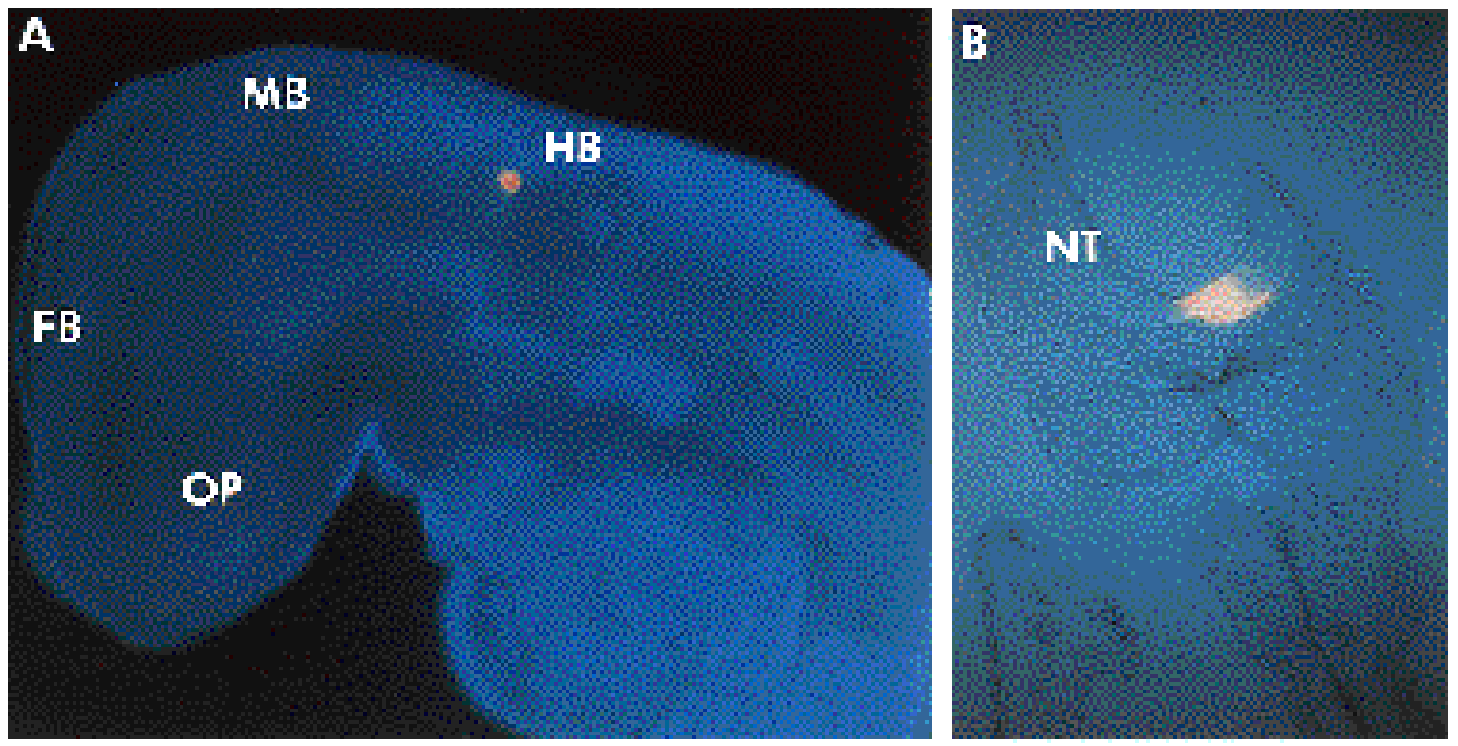

Fig. 7. DiI-injections into the ventral neural tube only label ventral neural tube cells. (A) A confocal image of an unoperated embryo that received a focal injection of DiI into the hindbrain at the level of caudal $\mathrm{r} 3$. After 36 hours, the labelled cells remained confined within a small region of the ventral neural tube. The optic cup (OP), forebrain (FB), midbrain (MB) and hindbrain (HB) are indicated for reference. (B) A transverse section through the same embryo illustrates that the DiI-labelled cells were confined to the ventral neural tube. Similar results were obtained for injections into the caudal midbrain or $\mathrm{r} 4$. 

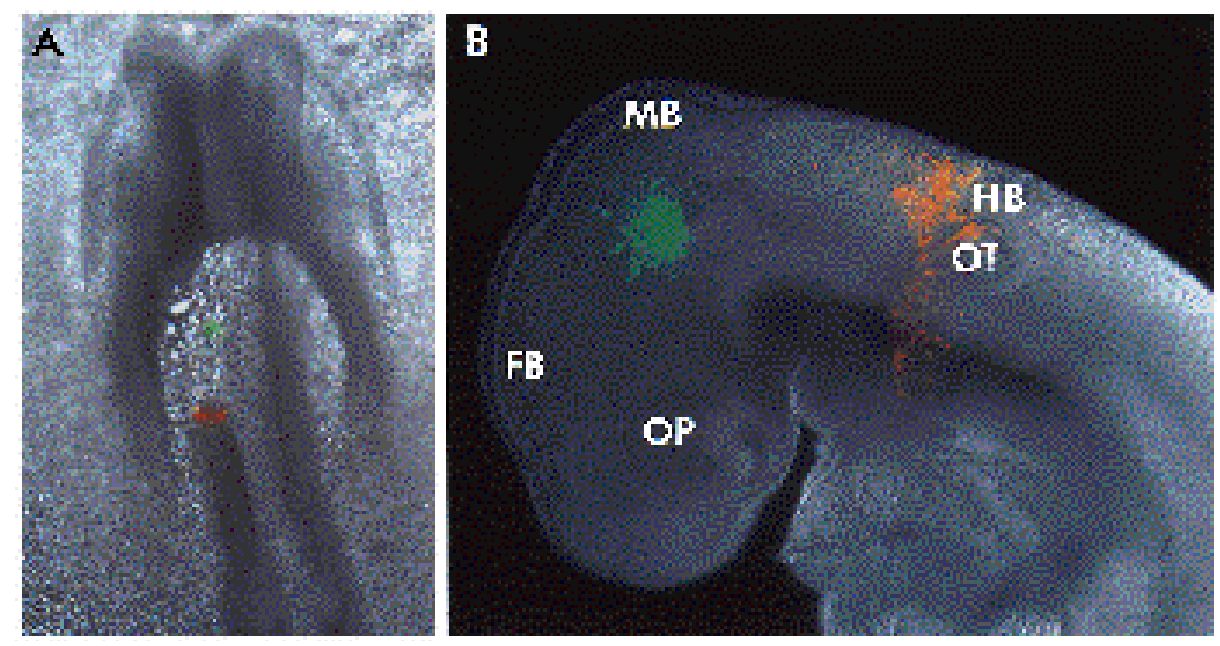

Fig. 8. Double-labelling with $\mathrm{DiO}$ and DiI illustrate that ablated regions and not the unoperated neural folds form neural crest after ablation.

(A) Immediately after a unilateral ablation of nearly half the neural tube, the embryo received a focal injection of $\mathrm{DiO}$ (green) into the residual neural tube in the midbrain, and DiI (reddishorange) into the bordering neural folds cells in the hindbrain. (B) After 36 hours, the neural tube cells (green) have dispersed to form neural crest cells in the midbrain. In contrast, the existing neural crest cells (reddishorange) have migrated laterally as expected for cells arising from $\mathrm{r} 4$, entering the second branchial arch. Only a few scattered cells deviated rostrally from their original trajectory. The optic cup (OP), forebrain (FB), midbrain (MB), hindbrain (HB) and otic vesicle (OT) are indicated for reference.

rostral or caudal neural crest bordering the cut edge or into the remaining fragment of the ventral neural tube. Transverse sections of these embryos 24 hours after ablation showed a closed neural tube about $1 / 3$ normal size (data not shown). After 36 hours, these embryos appeared morphologically normal. The patterns of DiI-labelled cells were virtually identical to those observed from unilateral ablations. The ventral neural tube appeared to reform the neural crest cells, such that DiI-labelled cells followed migratory patterns identical to neural crest cells in unoperated controls (Fig. 10). The one difference between bilateral and unilateral ablations was that there was a small amount of rostrocaudal compensation (less than $5 \%$ of total labelled cells) after extensive bilateral ablation in some embryos whereas less rostrocaudal compensation was observed after unilateral ablation.

\section{Long-term effects of unilateral neural tube ablation}

Embryos allowed to survive for 2-14 days following unilateral ablation of the neural tube to the ventral midline, excluding the floor plate, appeared to be morphologically normal except for occasional malformations in tissues of midbrain neural tube origin. For example, some minor abnormalities were noted in neural tube-derived structures such as the cerebellum and tectum, which appeared to have fewer neurons and axon tracts on the ablated than the control side in embryos analyzed 14 days postablation. Neural crest derived structures, such as the upper and lower beak, appeared normal (Fig. 3A). In addition, transverse sections through the trigeminal ganglion, which is partially derived from the neural crest, showed numerous neurofilamentimmunoreactive neurons; lightly DiI-labelled cells were observed in the trigeminal ganglia up to 3 days following the ablation. This suggests that the label on the cells is being diluted beyond detectability, by mitotic activity. There were no apparent differences in size of the ganglia or branchial arches on the operated and unoperated sides (Fig. 3B). In embryos observed 7 days postablation, the facial cartilage condensations (Fig. 11A,B) and the ciliary ganglia (Fig. $11 \mathrm{C}, \mathrm{D})$ appeared morphologically normal on both the ablated and control side.

\section{Loss of regulative ability}

All of the above experiments were performed on embryos at the 4-7 somite stage, prior to or concurrent with the onset of neural crest migration. Emigration of neural crest cells commences at the 6-7 somite stage in the midbrain and the 8-9 somite stage in the hindbrain (Lumsden et al., 1991; Sechrist et al., 1993). To determine the stages at which a complete regulation of the neural tube and neural crest is possible, we ablated half of the neural tube in the midbrain and/or hindbrain region of progressively older embryos. In 8 somite stage embryos (about 1.5 hours after the 7 somite stage), the labelled cells appeared to reform a complete neural tube; however, only occasional embryos had labelled cells within the cranial mesenchyme. By the 12 somite stage, labelled cells were observed in the neural tube, but never in the neural crest (Fig. 12). Thus, between the 8 and the 12 somite stage, the ability to reform neural crest cells diminished progressively. This suggests that the ability of neural tube cells to regulate to form neural crest cells ends 4.5-6 hours after the onset of neural crest emigration at the level of the midbrain and hindbrain.

\section{DISCUSSION}

The results presented above demonstrate that, in avian embryos prior to the 8 somite stage, sizable unilateral or bilateral deletions of the cranial neural tube fail to create a region of the embryo that is free of neural crest cells. The compensation for the missing neural crest must involve some remodelling of the remaining neural tube, since unilateral ablations approaching the ventral midline fail to eliminate either the neural tube or the corresponding region of neural crest. By labelling the cells bordering the ablation with DiI, it was possible to identify unambiguously the cells 

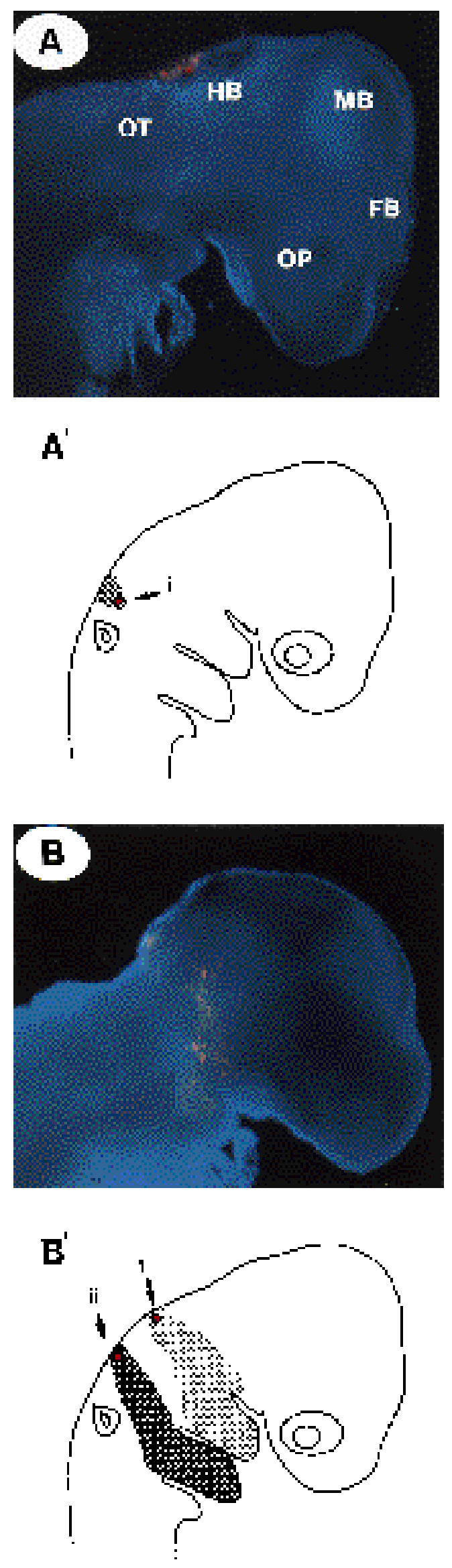

responsible for the reappearance of the neural crest. These experiments show that the neural tube cells ventral to the ablation, which normally would not form neural crest cells (Figs 5B, 7A, 9A) regulate to reform the missing regions of the neural tube and the neural crest after ablation of their dorsal neighbors (Figs 5C,D, 9B). Little if any of the compensation comes from neural crest cells rostral or caudal to the ablated zone (Figs 4C,D, 8); these cells migrate and proliferate as they would in unoperated embryos (Fig. 4A,B). Our results are in partial agreement with those of previous investigators (McGee and Ferguson, 1984) who showed that ablation of midbrain neural folds in chick embryos leads to normal development of neural crest-derived structures; although they suggested that neural crest cells rostral and caudal to the ablation were responsible for the compensation, the precise origin of the cells was not determined. The technique of DiI labelling of tissues adjacent to the ablated region permits assessment of the contribution of different regions to the neural crest and allows the labelled cells to be followed over time, thus providing a valuable adjunct to morphological data such as the scanning electron microscopic images used in the previous study (McKee and Ferguson, 1984).

The finding of such profound dorsoventral regulation in the cranial neural tube in the absence of significant rostrocaudal regulation may seem surprising, but is consistent with results from other studies of developmental potential and gene expression in the cranial neural tube. For example, cell lineage analysis at trunk levels shows that neural crest cells are not presegregated in the dorsal neural tube; instead, single precursors, injected with fluorescent dextran, commonly gave rise to descendants of both the neural crest and the neural tube (Bronner-Fraser and Fraser, 1988, 1989). Preliminary results suggest that similar behavior occurs in the cranial region. Thus, rostrocaudal patterning within the neural tube may be established well before dorsoventral patterning. By transplanting rhombomeres to different axial positions, Guthrie and colleagues (1992) have found that the pattern of Hox gene expression in the hindbrain caudal to rhombomere 3 is fixed by the 6 somite stage. Recently, expression of several genes containing a homeobox motif, including Emx1, Emx2, Otxl and Otx2, has been observed in more anterior regions of the brain (Simeone et al., 1992). In particular, Otxl is expressed as early as embryonic day 7.5 day in the telencephalon, diencephalon and mesencephalon of mice (Simeone et al., 1992), one day prior to

Fig. 9. Hindbrain ventral neural tube forms neural crest after ablation of the cells dorsal to it. For each class of operation, both a photograph of a representative embryo and a schematic diagram (') summarizing at least six cases are depicted. (A) In unoperated embryos, a focal injection of DiI into the ventral hindbrain neural tube resulted in labelled cells that were confined to a small region within the ventral neural tube. The optic cup (OP), forebrain (FB), midbrain (MB), hindbrain (HB) and otic vesicle (OT) are indicated for reference. (B) Following deep unilateral ablations of the neural folds and much of the neural tube, DiI-labelled neural tube cells immediately ventral to the ablation migrated away from the neural tube. Similar to the pattern for normal neural crest cells (Fig. 4B'), DiI labelling at the level of rhombomere 2 resulted in cells that extended to the first arch, whereas those from the level of rhombomere $4 / 5$ invaded the second arch. 


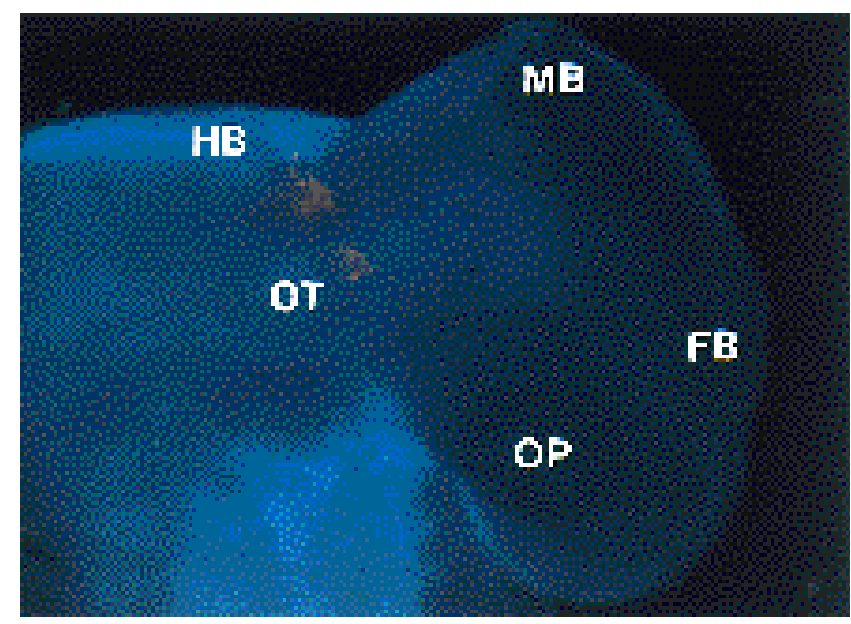

Fig. 10. The neural tube regulates to reform neural crest after bilateral ablation. The neural tube was removed, leaving only the ventral midline region, including the floor plate, which received a focal injection of DiI. 36 hours after ablation, DiI-labelled neural crest cells emerging from the remaining neural tube at the level of rhombomeres 4/5 were observed migrating toward the second branchial arch, as would normal neural crest cells from this axial level (compare with Fig. 4B' ii). The optic cup (OP), forebrain (FB), midbrain (MB), hindbrain (HB) and otic vesicle (OT) are indicated for reference.

the onset of neural crest migration (Serbedzija et al., 1992). In contrast, the present results suggest that dorsoventral commitment to a neural tube versus neural crest fate is not fixed until after the 8 somite stage. Several genes have been described which have selective patterns of dorsoventral localization in the cranial neural tube, including Wnts
(Dickinson and McMahon, 1992; Nusse and Varmus, 1992) and Pax genes (Gruss and Walther, 1992). As the molecular cascade of dorsoventral specification is elucidated, these and other transcription factors should provide powerful tools for determining the molecular mechanisms of the regulation reported here.

Based on the stereotyped patterns of migration and limited intermixing of neural crest cells, others have proposed that these cells have a rostrocaudal regional identity imprinted prior to their emigration (Noden, 1991; Hunt et al., 1991; Lumsden et al., 1991). For example, transplantation of the neural folds, whose cells normally populate the first branchial, to the level of the second branchial arch, results in the formation of first arch structures in place of the second arch including a duplication of the mandibles (Noden, 1983). In agreement with the proposal that the segmental origin of neural crest cells guides craniofacial patterning, a number of homeobox genes have been described with selective patterns of localization in the hindbrain (Hunt et al., 1991). However, it remains unclear if the pattern of expression of these transcription factors is fixed within the neural crest cells from the time they leave the neural tube. Because it remains possible that the expression of these genes in neural crest cells is conditional rather than autonomous, conclusions regarding a strict lineal assignment of axial addresses onto these cells must remain tentative. In addition to the hindbrain, some transcription factors have segmental patterns of expression in the forebrain and midbrain (Simeone et al., 1992), though their relationship to neural crest cells and craniofacial patterning has not been established.

There are significant differences between populations of neural crest cells along the neural axis. For example, cranial and trunk neural crest cells differ in developmental potential
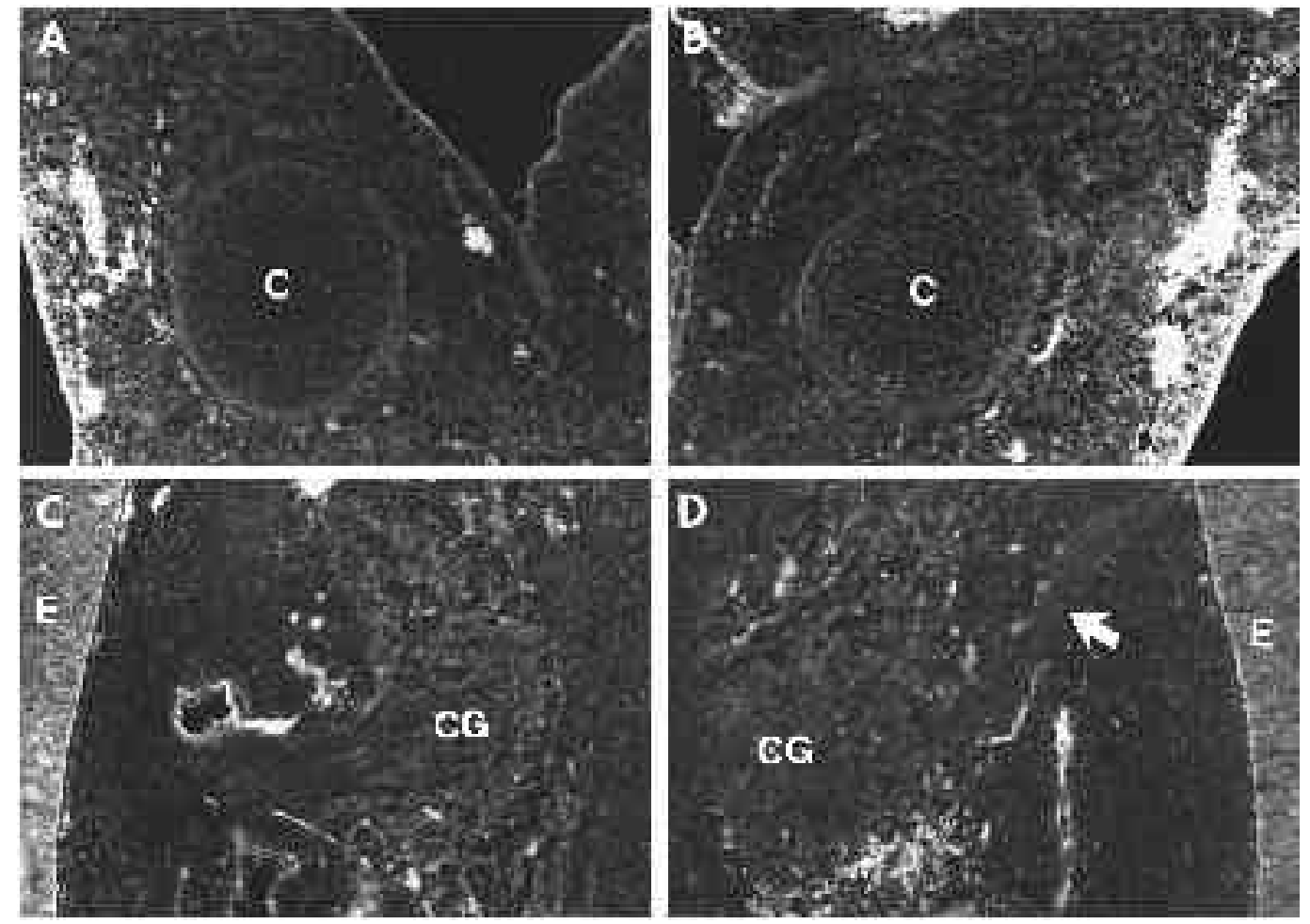

Fig. 11. Neural crest derivatives appear histologically normal on control and ablated sides of embryos 7 days postablation. Transverse sections through embryos showing neural crestderived facial cartilage and ciliary ganglia. (A,B) The cartilage condensations (C) appeared morphologically identical on the control (left) and ablated (right) sides within the frontonasal process of the face. (C,D) Similarly, the ciliary ganglia $(\mathrm{CG})$ were of proper size and in the correct position relative to the eye (E) on both the control (left ) and ablated (right) sides. Arrow indicates the ciliary nerve. 


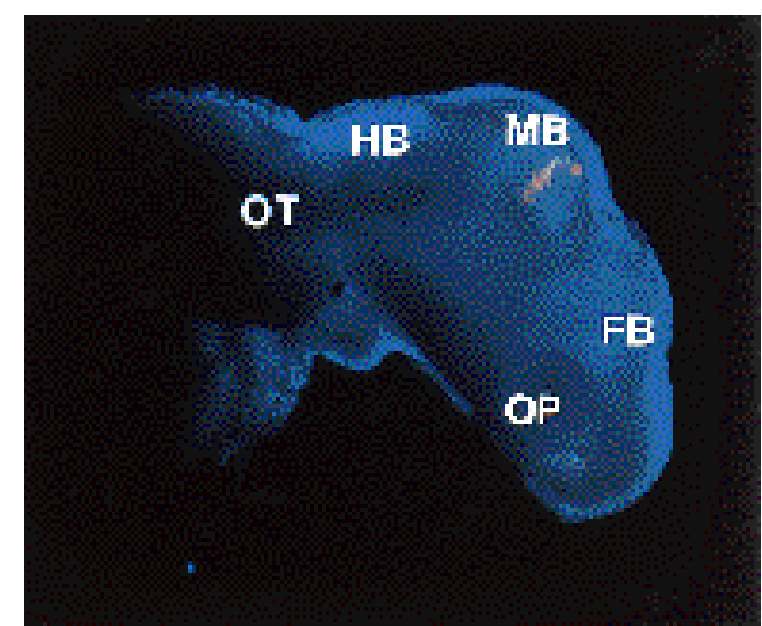

Fig. 12. Neural tube cells no longer regulate to form neural crest cells after the 8 to 12 somite stage. A 12 somite stage embryo in which the neural tube was ablated unilaterally at the level of the hindbrain and the adjoining neural tube immediately ventral to the ablations was labelled with DiI. The labelled cells proliferated and participated in formation of an apparently normal neural tube. However, no labelled cells had emigrated from the neural tube.

such that cranial neural crest cells form cartilaginous derivatives, whereas trunk neural crest cells cannot (LeDouarin, 1982). Comparison of our data with those of earlier investigators, suggest that there are axial differences in the regulative capacity of the neural tube/neural crest. In cranial regions, ablation of the neural folds results in reformation of the cranial neural crest from a regulation of the residual neural tube, rather than from some compensation by the neural folds more rostral or caudal. In contrast, similar removals in the trunk have been reported to result in some compensation by neural crest cells rostral and/or caudal to the ablated region, which can fill in up to three segments of neural crest cells (Yntema and Hammond, 1945, 1947). In the vagal region, there is apparently no regulation after removal of the cardiac neural crest (somitic level 1-3), resulting in the absence of the cardiac septum and the lack of division in the outflow tracts of the heart (Kirby et al., 1983). While conclusions regarding the presence or nature of these axial differences must await parallel experiments using techniques similar to those employed here, it is possible that the differences result from factors extrinsic to the neural tube. For example, the ability of the notochord to induce ventral properties in the neural tube (van Straaten et al., 1988; Yamada et al., 1991) might vary with axial position, thereby permitting or prohibiting regulation by the more ventral neural tube. Alternatively, the difference might result from factors intrinsic to the neural tube, such as differences in the proliferative ability of the neural tube at cranial, trunk and vagal levels.

The profound regulation observed in these studies immediately raises the question of how the potential to become neural crest is controlled in the normal embryo. Because both the dorsal and the ventral portions of the neural tube can respond to ablation of the neural folds, clearly there is no strict presegregation of 'crestogenic' potential. A variety of mechanisms might explain the assignment of some cells to become neural crest in both the regenerated and normal neural tubes. For example, all neural tube cells may have 'crestogenic' potential but some may be inhibited from the neural crest fate by interactions with the adjacent neuroepithelium. In this scenario, only those cells at the border of the neural tube would escape the inhibition and realize their potential to become neural crest. Alternatively, the ability of the neural tube to generate neural crest cells may be a derived fate, requiring interactions with other tissues such as the surface ectoderm. In this scenario, only those cells at the dorsum of the neural tube would be in a position to contact the ectoderm where they may be induced to give rise to the neural crest. Experiments in the axolotl show that ectoderm grafted to ectopic regions within any portion of the neural plate results in the formation of neural crest at the interface (Moury and Jacobson, 1990). As such grafts not only confront lateral ectoderm and neurectoderm but also create a discontinuity in the neurectoderm, they cannot distinguish between the two schemes outlined above.

The regulative response after unilateral ablation of up to half the neural tube must involve extensive proliferation to compensate for the missing tissue. Accordingly, increased numbers of mitotic cells have been reported following such surgeries (McKee and Ferguson, 1984). However, the DiIlabelled neural crest cells appear to arise from the cut edge prior to reclosure of the neural tube and without an obvious early increase in mitotic activity. Later, the neural tube reforms to attain comparable size to that in unoperated controls. It is possible that regulation of neural tube size may be a relatively late event, which involves increased cell survival rather than increased mitosis. Alternatively, subtle increases in cell cycle time may result in gradual restoration of neural tube size. The ability of the neural tube to reconstitute itself persists much longer than its ability to compensate for the loss of the neural crest. Ablations performed after the 9-12 somite stage result in a relatively normal appearing DiI-labelled neural tube, but no labelled neural crest cells. There are various factors that might contribute to the time-related changes in the regulative ability of the neural tube. Some possible explanations are that, (1) neural tube cells become committed to neural tube fates, perhaps by interactions with the underlying notochord, which prohibits them from regenerating the neural crest, (2) the neural crest cells are regenerated, but are prohibited from emigrating from the neural tube by environmental cues, (3) the ectoderm and the neurectoderm no longer interact effectively to promote cells to the 'crestogenic' state, perhaps due to the loss of either a signal from the ectoderm, or the ability of the neurectoderm to respond to a signal. Grafting and culture experiments, which combine tissues of different age and origin, should permit these and other possibilities to be tested.

Our results demonstrate that the cranial neural tube has a remarkable capacity to regulate and reform the neural crest following either minor or major ablations. The regenerative response appears to involve only neural tube cells at the same axial level as the ablation. The absence of any sizable contribution from the more rostral and caudal neural fold cells raises the interesting possibility that this response is constrained by regional identities that specify rostrocaudal position along the neuraxis. The reformation of the neural 
crest appears to be possible during a relatively short period of time, and is lost at or near the time of normal neural crest cell emigration. Thus, several factors may converge to dictate which cells become a part of the neural crest, including their position along the neuraxis, their position relative to the epidermis or notochord and their time of origin. Clearly, the same factors may have important consequences for establishing the developmental potential, migratory patterns and eventual fates of these cells.

We thank Dr John Sechrist helpful comments, suggestions and technical input, Dr Mark Selleck for helpful comments on the manuscript and Simone Lutolf for assistance with cryosectioning. This work was supported by USPHS grant HD-25138 to M. B.-F. and HD-29304 to S. E. F.

\section{REFERENCES}

Bronner-Fraser, M. and Fraser, S. (1988). Cell lineage analysis shows multipotentiality of some avian neural crest cells. Nature 335, 161-164.

Bronner-Fraser, M. and Fraser, S. (1989). Developmental potential of avian trunk neural crest cells in situ. Neuron 3, 755-766.

Dickinson, M. E. and McMahon, A. P. (1992). The role of Wnt genes in vertebrate development. Curr. Opin. Genet. Dev. 2, 562-566.

Gruss, P. and Walther, C. (1992). Pax in Development. Cell 69, 719-722.

Guthrie, S., Muchamore, I, Kuroiwa, A., Marshall, H., Krumlauf, R. and Lumsden, A. (1992). Neuroectodermal autonomy of Hox-2.9 expression revealed by rhombomere transpositions. Nature 356, 157-159.

Hamburger, V. and Hamilton, H. L. (1951). A series of normal stages in the development of the chick embryo. J. Morph. 88, 49-92.

Hunt, P., Wilkinson, D. and Krumlauf, R. (1991). Patterning the vertebrate head: murine Hox 2 genes mark distinct subpopulations of premigratory and migrating cranial neural crest. Development 112, 43-50.

Kirby, M. L. (1989). Plasticity and predetermination of mesencephalic and trunk neural crest transplanted into the region of the cardiac neural crest. Dev. Biol. 134, 402-412.

Kirby, M. L., Gale, T. F. and Stewart, D. E. (1983). Neural crest cells contribute to aorticopulmonary septation. Science 220, 1059-1061.
LeDouarin, N. M. (1982). The Neural Crest. London: Cambridge University Press.

LeDouarin, N. M. and Teillet, M. A. (1974). Experimental analysis of the migration and differentiation of neuroblasts of the autonomic nervous system and of neuroectodermal mesenchymal derivatives, using a biological cell marking technique. Dev. Biol. 41, 162-184.

Lumsden, A., Sprawson, N. and Graham, A. (1991). Segmental origin and migration of neural crest cells in the hindbrain region of the chick embryo. Development 113, 1281-1291.

McKee, G. J. and Ferguson, M. W. J. (1984). The effects of mesencephalic neural crest cell extirpation on the development of chicken embryos. J. Anat. 139, 491-512.

Moury, J. D. and Jacobson, A. G. (1990). The origins of neural crest cells in the axolotl. Dev. Biol. 141, 242-253.

Noden, D. M. (1983). The role of the neural crest in patterning of avian cranial skeletal, connective and muscle tissues. Dev. Biol. 96, 144-165.

Noden, D. M. (1991). Vertebrate craniofacial development: the relation between ontogenetic process and morphological outcome. Brain Behav. Evol. 38, 190-225.

Nusse, R. and Varmus, H. E. (1992). Wnt Genes. Cell 69, 1073-1087.

Sechrist, J., Serbedzija, G. N., Scherson, T., Fraser, S. E. and BronnerFraser, M. (1993). Segmental migration of the hindbrain neural crest does not arise from its segmental generation. Development 118, xxx-xxx.

Serbedzija, G., Bronner-Fraser, M. and Fraser, S. E. (1992). Vital dye analysis of cranial neural crest cell migration in the mouse embryo. Development 116, 297-307.

Simeone, A., Acampora, D., Gulisano, M., Stornaiuolo, A., Boncinelli, E. (1992). Nested expression domains of four homeobox genes in developing rostral brain. Nature 358, 687-690.

van Straaten, H. W. M., Hekking, E. J. L. M., Wiertz-Hoessels, F. T., Drukker, J. (1988). Effects of the notochord on the differentiation of a floor plate area in the neural tube of the chick embryo. Anat. Embryol. 177, 317-324.

Yamada, T., Placzek, M., Tanaka, H., Dodd, J. and Jessell, T. M. (1991). Control of cell pattern in the developing nervous system: Polarizing activity of the floor plate and notochord. Cell 64, 635-647.

Yntema, C. L. and Hammond, W. S. (1945). Depletions and abnormalities in the cervical sympathetic system of the chick following extirpation of the neural crest. J. Exp. Zool. 100, 237-263.

Yntema, C. L. and Hammond, W. S. (1947). The development of the autonomic nervous system. Biol. Rev. 22, 344-357.

(Accepted 30 April 1993) 
1062 T. Scherson and others 